\title{
SEDENTARISMO EN NIÑOS COSTARRICENSES: SI HAY SOLUCIÓN
}

Carlos Álvarez Bogantes

Escuela Ciencias del Deporte, Facultad de Ciencias de la Salud, Universidad Nacional calvarez@una.ac.cr

\section{RESUMEN}

El propósito de este artículo fue el de revisar la naturaleza de los patrones de movimiento de los niños, con el fin de entrelazarlos con las recomendaciones que se han establecido en cuanto a la actividad física que deben realizar y compararlas con las que tradicionalmente, se han seguido en el entorno costarricense. En una segunda parte del artículo se menciona al juego como la alternativa para rescatar el disfrute de moverse en los niños y de poder propiciar estilos de vida activa usando como elemento precursor las clases de educación física.

PALABRAS CLAVES: actividad física, patrones de movimiento, juego, educación física.

\section{SEDENTARISM IN COSTA RICAN CHILDREN: YES, THERE IS A SOLUTION}

\begin{abstract}
The purpose of this article was to review the nature of physical activity patterns of children in order to relate them to the recommendations provided regarding the physical activity that children must have. Additionally, these recommendations are compared to what has been done traditionally in Costa Rica. In another section of the article, games are mentioned as an alternative for making movement fun for children and creating active lifestyles mainly using physical education classes.
\end{abstract}

KEY WORDS: physical activity, movement patterns, games, physical education.

\section{INTRODUCCIÓN}

Hoy más que nunca, se hace necesario que los profesionales en salud y educación física conozcan la cantidad y tipo de actividad física que requieren los niños costarricenses, con el fin de que las acciones e intervenciones a desarrollar en estas edades, puedan coadyuvar a paliar el hecho que muestra que los niños costarricenses presentan estilos de vida sedentarios y niveles de obesidad alarmantes (Álvarez, 2003; Núñez, Monge, León y Rosello, 2003). Los hallazgos existentes de los niveles alarmantes de sedentarismo, sitúa a los niños costarricenses en predisposición a mantener la tendencia dentro de la vida adulta y a aumentar el riesgo de desarrollar enfermedades no transmisibles (Eptein et al, 1997), esto coloca a las instancias responsables en el campo, a considerar los elementos básicos del 
movimiento en los niños a la hora de diseñar e implementar estrategias educativas congruentes con el problema.

El propósito principal de este artículo es el de mostrar los elementos que se deben considerar a la hora de propiciar actividad física para la salud en los niños. Además, presenta al juego como herramienta básica para rescatar el gusto del niño por el movimiento, recomendándose su aplicación en las clases de educación física.

\section{Desarrollo}

Se ha demostrado que la enfermedad coronaria es un problema pediátrico, debido a que los factores de riesgo, especialmente el sedentarismo y la obesidad, que conducen a la aterosclerosis, comienzan en la niñez, por lo que el control temprano y adecuado de estos factores, mejora el pronóstico, por lo que las medidas preventivas en el sistema educativo costarricense deben iniciarse desde la infancia con el fin de eliminar, minimizar y controlar la enfermedad cardiovascular (Eptein et al, 1997).

\section{$\underline{\text { Características de movimiento en la niñez y en la vida adulta }}$}

Al tratar de entender las formas habituales de movimiento en estudiantes de primaria, se debe partir del hecho que ellos presentan la inclinación de ser más activos que la población adulta, por lo que la tendencia es que mantengan niveles apropiados de aptitud física (ACSM, 1995). Sin embargo, cierta evidencia inclina la balanza a pensar que la población estudiantil de primaria, en nuestro medio, podría estar desarrollando estilos de vida inactivos que atenten a futuro contra los niveles apropiados de aptitud física que ellos tienen (Álvarez et al, 2001; Fernández et al, 1996; Núñez, et al, 2001).

Algunas investigaciones en el plano internacional, sugieren una base biológica para explicar la diferencia entre los patrones de movimiento a edades tempranas en comparación a los de los adultos (Rowland, 1998). Primariamente, los niños y las niñas son seres vivos que juegan físicamente en forma incesante. Además, ellos juegan para con el objeto de recibir el estímulo necesario para su desarrollo, pero más importante, el movimiento propiciado por el juego brinda al sistema nervioso el estímulo primario para lograr un desarrollo armonioso. Por el contrario, los adultos presentan patrones de actividades altamente estructuradas y organizadas (Pancrazi et al, 1996). Cuando a los niños y niñas se les da la oportunidad para moverse, ellos demostrarán que son el segmento más activo de la población (Rowland et al, 1997). Esto nos da la posibilidad de afirmar, que esta población es biológicamente activa, y que cualquier otro comportamiento encontrado debe ser fuertemente contrarrestado, para volver al equilibrio corporal. En el caso de los adultos, ellos tienen infinidad de actividades que son de una índole muy variada, por lo que el estímulo que el sistema nervioso central necesita comprende algunas actividades activas, pero preferentemente de naturaleza pasivas, como por ejemplo: la lectura, coser, pintar, etc. (Wright, 2000).

Otra de las diferencias entre la población infantil y los adultos se refiere al volumen de movimiento que los niños y niñas desarrollan a través de todo el día. Debido al hecho de que los adultos disponen de menor tiempo libre, ellos optan por actividades de alta intensidad y baja duración (Corbin et al, 1996; ACSM, 1995). Los niños y las niñas carecen de las restricciones de tiempo en la mayoría de los casos, por lo que cuando tienen las condiciones ambientales apropiadas, ellos acumularán volúmenes más altos de movimiento, en forma espontánea. Como otra característica en el movimiento a edades tempranas, se debe mencionar que se presentan patrones esporádicos e intermitentes de desplazamiento físico, y esto obedece a que tienen una tolerancia limitada a la actividad física vigorosa. Además, presentan una relación muy baja entre aptitud física y actividad física, lo que lleva a descartar la conducta activa como una forma de determinar una buena aptitud física (Pate et al, 1996). 
Autores como Payne y Morrow (1995) han mostrado que las ganancias en la aptitud cardio-respiratoria son reducidas en los niños; lo que lleva a establecer que en estas edades la respuesta del entrenamiento es reducida en comparación con los adolescentes. Actividades consideradas en los adultos apropiadas para aumentar significativamente la capacidad cardio-respiratoria, no lo logran en los niños, lo cuál ha descartado el uso de las mediciones aeróbicas en los niños y niñas. Usualmente, los profesionales en educación física que conducen programas de mejoramiento de la condición aeróbica, esperan el mejoramiento de esta condición en los estudiantes, y al no obtenerlo ponen mayor presión, tratando de obtener resultados. O lo que es lo peor, esperan que la aptitud cardiovascular mejore, sin la intervención apropiada (Álvarez, 2001).

\section{$\underline{\text { Recomendaciones de actividad física en niños }}$}

La validez de realizar actividad física 3 veces por semana, durante 20 o 60 minutos, a una intensidad de $60 \%$ a $90 \%$ de la capacidad máxima, ya no es aceptada cuando se trabaja con niños. En la actualidad, el concepto es estimular a los niños a acumular de 30 a 60 minutos diariamente de actividad física, en forma discontinua, sumando todos los momentos de movimiento de los niños. El tipo de actividad que incluye esta nueva recomendación, va desde caminar de la escuela a la casa y viceversa; ayudar en la casa; manejar la bicicleta; saltar la cuerda en los recesos; ir a la pulpería; o pasear la mascota La meta debe ser que los niños acumulen 60 minutos de actividad física diariamente. Estos episodios de actividad física pueden estar divididos de 6 períodos de diez minutos cada uno o cuatro de 15 minutos o cualquier otra división que sea posible (Corbin et al, 1996).

Es importante tomar en cuenta que la actividad de alta intensidad no necesita ser mantenida por largos periodos de tiempo. Pequeños incrementos en la actividad física, de baja a moderada, pueden proveer los beneficios en salud y desarrollar la motivación para continuar una vida activa (Corbin et al, 1996).

Las recomendaciones anteriores están basadas en el hecho de que los niños si se les provee con un entorno que estimule el movimiento, ellos serán constantemente activos usando el juego. Aunque en nuestra sociedad los niños cada vez tienen menor cantidad de tiempo y oportunidades para moverse, ellos siguen siendo la porción más activa de nuestra sociedad, circunstancia que se debe luchar por mantener, estimulándolos para que realicen actividad física de moderada intensidad en forma discontinua. Conforme ellos juegan, se alternan episodios de movimiento con momentos de descanso, lo cuál es el proceso normal de los niños. La vieja costumbre de someter a los niños a actividades continuas y de alta intensidad debería ser cosa del pasado en el entorno educativo y volcarnos a comprender que este tipo de actividades no responden a las necesidades de los niños, siendo generadoras en muchas circunstancias de rechazo por ellos. Adicionalmente, se debe considerar que si se les provee con la posibilidad los niños serán activos, siempre que se recuerde que ellos son intermitentes en sus estructuras de movimiento. Esto último debe calar hondo en todos los que trabajen con niños con el objeto de que no los sometan a actividades o pruebas de larga duración, incluso si estas son de baja intensidad, ya que a cortas edades los niños no sienten atracción hacia actividades de alta intensidad (Corbin et al, 1996).

Cuando los adultos conociendo lo anterior, lo dejan de lado, se podría estar gestando las bases del rechazo futuro hacia la actividad física (Álvarez, 2003). Adicionalmente, todo educador físico debe considerar el hecho de los niños no continuarán una actividad si no encuentra razones concretas para continuar. A estas edades los niños no tienen una capacidad de pensamiento abstracto, lo que dificulta la transmisión de los conceptos abstractos de la importancia de la educación física, ellos sólo desean moverse. 


\section{$\underline{\text { El Juego }}$}

Para lograr el objetivo de propiciar el aumento de la actividad física, se debe rescatar el juego como la herramienta natural para lograr el estímulo para un desarrollo equilibrado. Es a través del juego que el niño tiene la posibilidad de recobrar el gusto por el aprendizaje, en un ambiente natural que le ofrece disfrute. Cuando los niños se sumergen en el juego no solo están aprendiendo de su mundo, pero también están llenando una necesidad sicológica de enriquecer el desarrollo de la inteligencia y de un sentido único de felicidad (Harrell et al, (1998). Autores como Piaget, Lorenz y Erickson (1998) establecen que el juego representa un aspecto esencial en el desarrollo del niño, ya que está ligado al desarrollo del conocimiento, de los aspectos afectivos, de la motricidad, de la socialización, en pocas palabras el juego es la vida misma del niño. En el juego el niño encuentra el éxito que muchas veces el deporte y del ejercicio les niega, lo que permite que la adherencia a la actividad se vea truncada (Douthitt, 1994). Adicionalmente, algunos autores han reportado que el disfrute de la actividad en el niño es la principal dimensión de la actitud del individuo asociada a la intención de participar. Pero es cuando el movimiento es placentero, que este brindará un sentimiento de sí puedo, que podría aumentar la participación en la actividad física (Corbin et al, 1996).

Aunque las palabras disfrute, diversión y entretenimiento son difíciles de definir, hay que rescatarlas y hacerlas parte de la vida cotidiana de los niños escolares costarricenses. Huizinga (1970), autor de la idea de que el juego es la fuerza de la civilización en la experiencia humana, escribió que la idea de jugar resiste todo análisis y toda lógica de interpretación. El gozo que otorga el juego y el movimiento, es generalmente asociado con un sentimiento de felicidad en el niño (Henderson et al, 1999). En este medio la gente asocia este gozo con el tiempo libre y la recreación, aunque pueden presentarse ambas sin disfrute.

\section{Educación física}

Que mejor lugar que la clase de educación física para recuperar la alegría de aprender a través del juego. La actividad física en los centros educativos debe rescatar los elementos positivos de la actividad física, como son; el disfrute, el amor, orgullo, la emoción, el reto, el valor, el cariño, la confianza, y muchas otras condiciones de la experiencia personal. Muchas veces las clases de educación física se transforman en una clase más del currículo, en donde lo importante es la organización excesiva y la medición, dando como resultado la pérdida del disfrute para la mayoría (Henderson et al, 1999). Como resultado del divorcio entre disfrute y educación, muchos niños han olvidado la alegría que otorga el movimiento.

Los padres y los maestros, deben entender que los niños pueden perder el entusiasmo por los asuntos académicos o que el mismo sistema educativo les puede "matar" el entusiasmo y curiosidad a los niños, si son sometidos a procesos monótonos, autoritarios y aburridos. Todo ser humano usualmente disfruta basado en la libre elección y en la motivación intrínseca. Cuando esos dos elementos desaparecen del contexto, el resultado es el niño mecánico y autómata, que vemos en muchas de las escuelas de nuestro país. Si el maestro no provee la posibilidad de escoger, eliminar la competencia, estimular la creatividad y la espontaneidad, y los alumnos deciden no comprometer su energía mental, física y emocional en la actividad, dudosamente el disfrute sucederá (Malina, 1996). Para poder disfrutar es necesario involucrar elementos mentales y cognoscitivos como: fijarse metas inmediatas, estar en un ambiente que propicie la actividad y que brinde retroalimentación en los niveles de éxito. Además, los elementos de sensación de fluidez en lo que se realiza, sentimiento de control y de dominio sobre la actividad, contribuyen al placer derivado de la actividad física y el juego (Henderson et al, 1999). 
A diferencia de los adultos, que presentan niveles de actividad muy estructurados, organizados y limitados, los niños cuando se les ofrece la posibilidad de jugar, ellos jugarán. Los educadores físicos deben estar conscientes que ellos forman la persona integralmente y que la actividad física y el juego impactan la parte cognitiva, sicomotor y afectiva. Si el sistema educativo desea estimular estilos de vida saludable, se debe tomar en cuenta que los niños deben recuperar el disfrute del movimiento. Las estrategias que los profesionales del movimiento podrían utilizar para promover la actividad física y otros estilos de vida saludables, deben estar basadas en ayudar a otros a ver y sentir el movimiento como una forma valiosa de disfrutar. El concepto de que los niños son adultos en cuerpos pequeños, que pueden ser sometidos a la rigurosidad del entrenamiento debe ser desterrado de los centros educativos. Es común ver educadores, formados para el rendimiento, someter a sus alumnos a actividades físicas como si se estuviera formando atletas. Los niños, según Pangrazi et al, (1996), establecen que los niños son esporádicos en sus patrones de movimiento, por lo que requieren actividades lúdicas que contemplen esta situación.

Como resumen, se recomienda la actividad física intermitente y de moderada intensidad en los niños. Esta actividad física puede ser entre treinta a una ejecutada en forma continua o sumando toda la actividad que realiza el niño durante el día. Además, se presenta al juego como la herramienta natural del niño para que logre acumular la actividad necesaria para el desarrollo, pero sobre todo para logre recobrar el disfrute por la actividad física.

\section{Bibliografía}

ACMS. (1995). ACMS guidelines for exercise testing and prescription $\left(5^{\text {th }}\right.$ ed.). Baltimore: Williams and Wilkias.

Álvarez, (2001). Teoría transteorética: aplicación en estudiantes de secundaria. Documento manuscrito.

Álvarez, C. (2001). Valoración de la actividad física en niños costarricenses. Sin publicar.

Álvarez, C. (2003). Del Juego a la Salud. Documento manuscrito.

Corbin, C., Pancrazi, R. and Welk, G. (1994). Toward an understanding of appropriate physical levels for youth. Physical Activity and Fitness Research Digest. 1, 1-8.

Corbin, C. and Pancrazi, R. (1996). How much physical activity is enough? JOPERD: 67, 33-39.

Council for Physical Education for Children. (1998). Physical activity for children: statement of guidelines. Reston, VA: NASPE Publications.

Douthitt, V. (1994). Psychological determinants of adolescent exercise adherence. Adolescence. 29, 711-723.

Epstein, L., Saelens, B., Myers, M. and Vito. D. (1997). Effects of decreasing sedentary behaviors on activity choice in obese children. Health Psychology. 16, 107-113.

Fernández A, Ulate G, Romero M, y Murillo S. (1996). Factores de riesgo cardiovascular en estudiantes de 17-19 años de la Universidad de Costa Rica. Documento Técnico, Facultad de Medicina, Universidad de Costa Rica. 
Harrell, S., Gansky, S., McMurray, R., Frauman, A., and Bradley, C. (1998). School based interventions improve heart health in children with multiple cardiovascular disease risk factors. Pediatrics. 102, 371-380.

Henderson, K., Glancy, M. and Little, S. (1999). Putting the fun into physical activity. JOPERD. 70, 43-49.

Huizinga, J. (1970). Homo Ludens: Madrid, Alianza Editorial, Madrid.

Malina, R. (1996). Tracking of physical activity and physical fitness across the lifespan. RQES. 67, 48-57.

Núñez, H., Monge, R., León, H., and Rosello, M. (2003). Prevalence of overweight and obesity among Costa Rica elementary School Children. Rev. Panam. Salud Pública. 13, 275-276.

Pancrazi, R., Corbin, C. and Welk, G. (1996). Physical activity for children and youth. JOPERD. $67,38-43$.

Pate, R., Baronowski, R, Dowda, M. y Trost, S. (1996). Tracking of physical activity in young children. Medicine and Science in Sports and Exercise. 28, 92-96.

Patterson, P. (2000). Reliability, validity, and methodological response to the assessment of physical activity via self-report. RQES. 71, 15-20.

Payne, W. and Morrow, J. (1995). Exercise and VO2 max in children. RQES. 64, 305-313.

Piaget, J., Lorenz, K. y Erickson, E. (1998). Juego y desarrollo. Barcelona: Editorial Crítica.

Rowland, T. (1998). The biological basis of physical activity. Medicine and Science in Sports and Exercise. 30, 392-399.

Simons-Morton, B., Parcel, G. O'Hara, N., Blair, S., and Pate, R. (1988). Health-related physical fitness in childhood. Annual Review of Public Health. 9, 404-425.

Shepard, R., (1994). Physical activity and child health. Sport Medicine. 1, 205-233.

Trost, S., Pate, R., Dowda, M., Saunders, R., Ward, D., and Felton, G. (1996). Gender differences in physical activity and determinants of physical activity in rural fifth grade children. Journal of School Health. 66, 145-150.

Wright, M., Patterson, D. and Cardinal B. (2000). Increasing children's physical activity. JOPERD. $71,26-30$.

Fecha de recepción: agosto del 2006.

Fecha de aceptación: octubre del 2006.

Fecha de publicación: 31 de diciembre del 2006. 